\title{
The Type Specimens of Mosquitoes (Diptera, Culicidae) Deposited in the Entomological Collection of the Instituto Oswaldo Cruz, Rio de Janeiro, Brazil
}

\author{
Verônica Marchon-Silva/ ${ }^{+}$, Ricardo Lourenço-de-Oliveira*, Magaly Dolsan de \\ Almeida, Adenildo da Silva-Vasconcelos, Jane Costa
}

\author{
Coleção Entomológica *Laboratório de Transmissores de Hematozoários, Departamento de Entomologia, \\ Instituto Oswaldo Cruz, Av. Brasil 4365, 21045-900 Rio de Janeiro, RJ, Brasil
}

\begin{abstract}
A list of type specimens of 100 mosquito species deposited in the Entomological collection of the Instituto Oswaldo Cruz is presented. It includes five holotypes belonging to the subfamily Anophelinae; 56 holotypes of Culicinae and two of Toxorhynchitinae. A lectotype is designated for Toxorhynchites fluminensis. The holotypes of six nominal species - Psorophora chiquitana, Psorophora circunflava, Psorophora melanota, Psorophora lanei, Wyeomyia brucei and Uranotaenia noctivaga - previously considered non existent or of unknown location were found in the collection.
\end{abstract}

Key words: Diptera - Culicidae - Anophelinae - Culicinae - Toxorhynchitinae - mosquito type specimens

The importance of collections as a basis for scientific research is unquestionable and their significance has been stressed by the International Code of Zoological Nomenclature (72 D) and by many authors, such as Lent (1972) and Kim (1976). The Entomological collection of the Instituto Oswaldo Cruz (IOC) is diversified and contains around one million specimens. The collection began in 1900 with insects collected during scientific expeditions undertaken by IOC researchers. The scientific expeditions aimed the capture of insects to determine their relation to human and animal diseases, hence the collection has a rich scientific and historical patrimony.

Mosquitoes have been the subject of research of several workers in the Institution, such as its founder, Oswaldo Cruz. These researchers were mostly interested in general parasitology and tropical diseases, but dozens of mosquito species were described by them, as well as by visitors working in the Institution, such as J Lane and N Cerqueira. Presently, mosquito type specimens are mainly deposited in a general collection, called the "Central collection", or in three special collections presently closed to new deposits and named in honor of entomologists from the Institution: Cesar Pinto, Adolpho Lutz and Costa Lima. The insect collections were under Costa Lima's responsibility until his death in 1964. Afterwards, Orlando V Ferreira

Work performed with support of PAPES-FIOCRUZ Project.

+Corresponding author. Fax: 55-21-

Received 11 October 1995

Accepted 9 April 1996 and Sebastião José de Oliveira have subsequently taken them under their responsibility.

Recently, a list of type specimens of triatomine bugs, the vectors of Chagas disease, deposited in IOC was published by Gonçalves et al. (1994). A list of the mosquito type specimens deposited in the collection of the Institution is presented in this paper. Some specimens were previously regarded as lost (Belkin et al. 1971, Knight \& Stone 1977).

Mosquito species names are listed in alphabetical order within an alphabetical arrangement of subfamilies and genera, as proposed by Belkin et al. (1971). Each specific name is followed by the author's name, year of publication, data on the type material and a condensed description of its taxonomic status.

Subfamily Anophelinae

Genus Anopheles Meigen

- albitarsis Lynch-Arribálzaga, 1878. Anopheles (Nyssorhynchus). Neotype female (6153), larval and pupal skins on slides (5578-5580); Baradero, Buenos Aires, Argentina; MG Rosa-Freitas coll., 09 Feb 1986. Costa Lima collection. Rosa-Freitas and Deane 1989: 289 (neotype desig.).

- argyritarsis ssp sawyeri Causey, Deane, Deane and Sampaio, 1943. Anopheles (Nyssorhynchus). Paratypes: 1 male and 1 female $(393,394)$; São Benedito, Ceará, Brazil. Lutz collection.

- braziliensis (Chagas, 1907). Anopheles (Nyssorhynchus). Syntypes: 6 females (5530); rio das Velhas, Lassance, Minas Gerais, Brazil; 11 Jul 1907. Costa Lima collection.

- cuyabensis (Neiva and Pinto, 1923). Anopheles (Nyssorhynchus). Lectotype wing mounted on slide (403) together with type of rondoni, marked 
Mato Grosso, C Pinto, remainder of adult apparently lost. Paralectotype female (2027), left wing on slide (2561), right midleg missing; west bank of Rio Cuiabá, Fazenda São João, Mato Grosso, Brazil; C Pinto coll., Jun 1922. Cesar Pinto collection. Synonym of Anopheles (Nyssorhynchus) triannulatus (Neiva and Pinto, 1922). Pinto 1939: 406 (syn.). Belkin et al. 1971: 7 (lectotype desig.)

- deaneorum Rosa-Freitas, 1989. Anopheles (Nyssorhynchus). Holotype female (6171); Palheta, Guajará-Mirim, Rondônia, Brazil; PS Souza coll., 30 Jul 1986. Paratypes: 4 males (6172$6174,6176)$ and 4 females $(6175,6177-6179)$; ibid. Paratypes: 3 males $(6182,6184,6185)$ and 3 females $(6180,6181,6183)$; Rio Branco, Acre, Brazil; R Lourenço-de-Oliveira coll., 07 and 09 Oct 1985. Costa Lima collection.

- evandroi Costa Lima, 1937. Anopheles (Anopheles). Holotype female (1280, vial 3040), wings on slide (3285); São Bento, RJ, Brazil; Evandro Chagas coll., Feb 1935. Costa Lima collection.

- galvaoi Causey, Deane and Deane, 1943. Anopheles (Nyssorhynchus). Paratypes: 1 male and 1 female (391, 392), reared from eggs of females caught in Rio Branco, Acre, Brazil. Lutz collection.

- lanei Galvão and Amaral, 1938. Anopheles (Nyssorhynchus). Paratypes: 1 female (1210), larval skin on slide (1208) and 1 male (1209), genitalia on slide (1211); Vila Emílio Ribas, Pref. Sanitária de Campos do Jordão, Serra da Mantiqueira, São Paulo, Brazil; Amaral coll., Mar 1938. Cesar Pinto collection. Paratypes: 1 male and 2 females ( 3838); ibid.; Amaral coll., 1938. Costa Lima collection.

- lutzii Cruz, 1901. Anopheles (Nyssorhynchus). Syntypes: 3 females (1965), wing on slide (2111); Lagoa Rodrigo de Freitas, Rio de Janeiro, Brazil. Costa Lima collection.

- mattogrossensis Lutz and Neiva, 1911. Anopheles (Anopheles). Holotype female (3530), very damaged specimen, all legs, both antennae and right wing missing; proboscis and left wing broken distally; Lagoa de Manicoré [Mandioré], Mato Grosso, Brazil; JC Diogo coll., Aug 1908. Costa Lima collection.

- minor Costa Lima, 1929. Anopheles (Anopheles). Syntypes: 2 females (vial 281), wings on slides (673, 890); Estrela, [Imbariê], RJ, Brazil; C Lima coll., 14 Jan 1929. Costa Lima collection.

- rachoui Galvão, 1952. Anopheles (Anopheles). Paratype female (5800); Paranaguá, Paraná, Brazil. Costa Lima collection.

- rondoni (Neiva and Pinto, 1922). Anopheles (Nyssorhynchus). Lectotype: the largest wing mounted on slide (403) together with the type of cuyabensis, marked Mato Grosso, C Pinto coll., remainder of adult apparently lost. Paralectotypes: 1 female (2028), collected on a steamboat on rio Paraguai, Ladário, Mato Grosso, Brazil. Cesar Pinto coll., Jun 1922; 1 female (2029), ibid.; wings and a hindlegs on slides of a female (2559, 2560), ibid.; 1 female (63), left midleg and right midtarsomeres II-V missing; rio Cuiabá, Mato Grosso, C Pinto coll., Jun 1922; 1 female (67), left midleg missing; rio São Lourenço, Mato Grosso. C Pinto coll., Jun 1922. Cesar Pinto collection. Belkin et al. 1971: 6 (lectotype desig.)

- squamifemur Antunes, 1937. Anopheles (Lophopodomyia). Holotype female (2298); Vegagrande, Restrepo, Meta, Colombia; PCA Antunes coll., 29 Mar 1935. Costa Lima collection.

- triannulatus (Neiva and Pinto, 1922). Anopheles (Nyssorhynchus). Holotype female (2026), left midtarsomeres IV, V missing; west bank of rio Cuiabá, Fazenda São João, Mato Grosso, Brazil; C Pinto coll., 1922. Cesar Pinto collection.

Subfamily Culicinae

Tribe Aedini

Genus Aedes Meigen

- aurivittatus Cerqueira, 1943. Aedes (Howardina). Holotype female (389); Florida, Santa Cruz, Bolivia; Serv. Nac. de Febre Amarela coll., Feb 1940. Lutz collection.

- pennai Antunes and Lane, 1938. Aedes (Ochlerotatus). Paratypes: 1 male and 1 female (3955), Cabreuva, São Paulo, Brazil; ARR coll., Apr 1937. Costa Lima collection.

\section{Genus Psorophora Robineau-Desvoidy}

- albigenu (Peryassú, 1908). Psorophora (Janthinosoma). Holotype female (3523), right foreleg and one hindleg missing; Chanaan, São Paulo, Brazil; 24 Mar 1906. Costa Lima collection.

- chiquitana Pinto, 1932. Psorophora (Grabhamia). Holotype female (163), right wing on slide (163); Los Naranjos, Bolivia; Olímpio da Fonseca coll., 1925. Paratype female (164), Serra da Piusa, Porto Esperança, Mato Grosso, Brazil; JCN Penido coll., 29 Jan 1925. Cesar Pinto collection. Synonym of Psorophora (Grabhamia) confinnis (Lynch-Arribálzaga, 1891). Lane 1953: 768 (syn.).

- circunflava Cerqueira, 1943. Psorophora (Janthinosoma). Holotype female (387); Axiamas, Caupolican, La Paz, Bolivia. Serv. Nac. de Febre Amarela coll., Dec 1941. Lutz collection. 
- dimidiata Cerqueira, 1943. Psorophora (Grabhamia). Holotype male (385); Lagunilhas, Valle Grande, Santa Cruz, Bolivia. Serv. Nac. de Febre Amarela coll., Feb 1940. Allotype female (384), ibid. Lutz collection.

- forceps Cerqueira, 1939. Psorophora (Janthinosoma). Holotype male (4404) and allotype female (4405); Jacarepaguá, Rio de Janeiro, Brazil; RC Shannon coll., Dec 1937. Costa Lima collection.

- genumaculata Cruz, 1907. Psorophora (Psorophora). Lectotype male (2582), damaged specimen, without head and genitalia; Santos, São Paulo, Brazil; Dec 1906. Lutz collection. Synonym of Psorophora (Psorophora) saeva Dyar and Knab, 1906. Lane 1951: 335 (syn.).

- lanei Shannon and Cerqueira, 1943. Psorophora (Janthinosoma). Holotype female (383); Maracaju, Mato Grosso, Brazil. RC Shannon coll., Dec 1937. Lutz collection.

- melanota Cerqueira, 1943. Psorophora (Janthinosoma). Holotype male (386); Ribeiralta, Vaca Diez, Bolivia. Serv. Nac de Febre Amarela coll., Dec 1939. Lutz collection.

Tribe Culicini

Genus Culex Linnaeus

- carcinoxenus Oliveira Castro, 1932. Culex (Culex). Lectotype male (1967, vial 996), genitalia on slide (4186); state of São Paulo, Brazil. Oliveira Castro coll. Paralectotype female (1967, vial 995); ibid. Costa Lima collection. Belkin et al. 1971: 21 (lectotype desig.).

- chrysothorax (Peryassú, 1908). Culex (Microculex). Lectotype male (2033), genitalia on slide (2331); Copacabana, Rio de Janeiro, Brazil; Oct 1907. Costa Lima collection. Belkin et al. 1971: 23 (lectotype desig.). Sirivanakarn 1983: 273 (nomen dubium).

- corniger Theobald, 1903. Culex (Culex). Paratype male (vial 321); Paraná, Brazil. A Lutz coll., 1902. Costa Lima collection.

- lugens Lutz, 1905. Culex (Melanoconion). Syntypes: 1 female and 1 male (2610); Lagoa, São Paulo, Brazil; 3 Feb 1904. Lutz collection.

- rabelloi Forattini and Sallum, 1987. Culex (Melanoconion). Paratypes: 2 males (6148, 6149), genitalia on slides (5573, 5574); Rio Jacaré-Pipira, Dourado, São Paulo, Brazil; Forattini coll., Sept-Oct 1980; 1 male (6150), pupal skin and genitalia on slide (5575); Cananeia, Itapirangi; Forattini coll., 28 Sept 1984; 1 male (6151), larval and pupal skins and genitalia on slide (5576); Pariquera-Açú, Estação Experimental; Forattini coll., Mar 1983; 1 male (6152), larval and pupal skins on slide (5577); lagoa Sabauna, Iguapé, São Paulo; Forattini coll., 10
Jul 1985. Costa Lima collection.

- ribeirensis Forattini and Sallum, 1985. Culex (Melanoconion). Paratypes: 5 males and 5 females (6113-6122), male genitalia on slides (5538-5542); Pariquera-Açú, São Paulo, Brazil; Forattini coll., Sept 1978 to Feb 1981. Costa Lima collection.

- siphanulatus Lourenço-de-Oliveira and Silva, 1987. Culex (Microculex). Holotype male (6128), genitalia, larval and pupal skins on slides (5551, 5552); Granjas Calábria, Jacarepaguá, Rio de Janeiro, Brazil; R Lourenço-de-Oliveira coll., 20 Dec 1982. Allotype female (6128), larval and pupal skins on slides (5553); ibid. Costa Lima collection. Paratypes: 4 females and 5 males $(338,357,358,378,457,461,462,466,470)$. Central collection.

Tribe Mansoniini

Genus Coquillettidia Dyar

- albicosta (Peryassú, 1908). Coquillettidia (Rhynchotaenia). Lectotype female (vial 430), damaged specimen, all legs missing; Xerém, RJ, Brazil; 28 Oct 1907. Costa Lima collection. Belkin et al. 1971: 16 (lectotype desig.).

- chrysonotum (Peryassú, 1922). Coquillettidia (Rhynchotaenia). Syntypes: 4 females (vials 431,432); Vale do Rio Doce, Espírito Santo, Brazil; Peryassú coll. Costa Lima collection.

- juxtamansonia (Chagas, 1907). Coquillettidia (Rhynchotaenia). Lectotype female (vial 428), wing on slide (1001); Juiz de Fora, Minas Gerais, Brazil; Arthur Neiva coll., Oct 1906. Costa Lima collection. Belkin et al. 1971: 16 (lectotype desig.).

- nitens (Cerqueira, 1943). Coquillettidia (Rhynchotaenia). Holotype female (388); Puerto Soarez, Santa Cruz, Bolivia; Serv. Nac. de Febre Amarela coll., Jul 1939. Lutz collection.

Genus Mansonia Blanchard

- chagasi (Costa Lima, 1935). Mansonia (Mansonia). Holotype male (1956); Bicudo, Minas Gerais, Brazil; C Chagas coll., 11 Feb 1908. Costa Lima collection.

- fonsecai Pinto, 1932. Mansonia (Mansonia). Holotype female (160), right foreleg and hindlegs missing; Los Naranjos, Bolivia; O da Fonseca coll., 1925. Paratypes: 2 females $(159,161)$, wing on slide (159); ibid. Cesar Pinto collection. Synonym of Mansonia (Mansonia) indubitans Dyar and Shannon, 1925. Costa Lima 1935: 467 (syn.).

Tribe Orthopodomyiini

Genus Orthopodomyia Theobald

- albicosta (Lutz, 1904). Orthopodomyia. Lecto- 
type female (2663), São Paulo, Brazil. Lutz collection. Belkin et al. 1971: 18 (lectotype desig.).

- sampaioi Costa Lima, 1935. Orthopodomyia. Lectotype male (1861, vial 942), genitalia in a capillary vial (1991), wings and antennae on slides (1992, 2089); Tijuca, Rio de Janeiro, Brazil; PC Sampaio coll., Jun 1934. Costa Lima collection. Zavortink 1968: 82 (lectotype desig.)

- townsendi Costa Lima, 1935. Orthopodomyia. Holotype female (582), foreleg and wing on slide (box 45); Rio Tapajós, Boa Vista, Pará, Brazil; CHT Townsend coll., 11 Out 1932. Cesar Pinto collection. Synonym of Orthopodomyia fascipes (Coquillett 1906). Lane 1951: 335 (syn.).

Tribe Sabethini

Genus Johnbelkinia Zavortink

- edwardsianus Lane and Cerqueira, 1942. Johnbelkinia. Holotype female (19744), right foretarsi and left hindleg missing; Belterra, Santarém, Pará, Brazil; Serv. Nac. de Febre Amarela coll., Sept 1938. Central collection. Synonym of Johnbelkinia longipes (Fabricius, 1805). Zavortink 1979: 35 (from genus Trichoprosopon; syn.)

\section{Genus Phoniomyia Theobald}

- bonnei Lane and Cerqueira, 1942. Phoniomyia. Holotype male, larval and pupal skins and genitalia on slides $(2395,2443)$, left foretarsi and left midleg missing; Rio de Janeiro, Brazil; L Whitman coll., 1940. Allotype female, larval and pupal skins on slide (2393), right mid- and forelegs missing; ibid. Central collection.

- davisi Lane and Cerqueira, 1942. Phoniomyia. Holotype male, genitalia on slide (1551), right foreleg missing; Mangaratiba, RJ, Brazil; RC Shannon coll., Jul 1938. Allotype female, ibid. Central collection.

- deanei Lourenço-de-Oliveira,1983. Phoniomyia. Holotype male (6123), larval and pupal skins and genitalia on slide (5543); Granjas Calábria, Jacarepaguá, Rio de Janeiro, Brazil; R Lourençode-Oliveira coll., 10 Dec 1982. Allotype female (6124), larval and pupal skins on slide (5544); ibid. Costa Lima collection. Paratypes: 5 females [larval and pupal skins on slides (526-530)]; 7 males [larval and pupal skins on slides (531537)]. Central collection.

- edwardsi Lane and Cerqueira, 1942. Phoniomyia. Paralectotype [= allotype] female, larval and pupal skins on slide (2478); Rio de Janeiro, Brazil; L Whitman coll., 1940. Central collection. Belkin et al. 1971: 12 (lectotype in USNM).

- flabellata Lane and Cerqueira, 1942. Phoniomyia. Holotype male, genitalia on slide (1904), left hind- and right midtibiae and tarsi missing; Muriqueira, Bahia, Brazil; Rockfeller Foundation Lab., RC Shannon coll., 26 Apr 1929. Allotype female, right wing missing; ibid. Central collection.

- neivai Lane and Cerqueira, 1942. Phoniomyia. Holotype female (12547), right hindleg missing; Londrina, Paraná, Brazil; Serv. Nac. de Febre Amarela coll., Feb 1937. Central collection. Synonym of Phoniomyia quasilongirostris Theobald, 1907. Correa and Ramalho 1956: 50 (syn.).

- palmata Lane and Cerqueira, 1942. Phoniomyia. Holotype male, larval and pupal skins, hindlegs and genitalia on slide $(2436,2444)$; right midleg missing; Rio de Janeiro, Brazil; L Whitman coll., 1940. Allotype female, pupal skin on slide (2434), right mid- and hindlegs missing; ibid. Central collection.

- theobaldi Lane and Cerqueira, 1942. Phoniomyia. Holotype male, genitalia on slide (3011), right fore-, mid- and hindlegs missing; Rio de Janeiro, Brazil; Serv. Nac. de Febre Amarela coll., Mar 1938. Central collection.

Genus Runchomyia Theobald

- reversa (Lane and Cerqueira, 1942). Runchomyia (Runchomyia). Holotype male, larval and pupal skins, and genitalia on slides (2945, 2946); Magé, RJ, Brazil. RC Shannon coll., Jul 1940. Allotype female; larval and pupal skins on slide (2947), right foreleg missing; ibid. Central collection. Zavortink 1979: 12 (from genus Trichoprosopon).

- theobaldi (Lane and Cerqueira, 1942). Runchomyia (Runchomyia). Holotype male, larval and pupal skins and genitalia on slides (3002, 3007); Magé, RJ, Brazil; RC Shannon coll., 22 May 1940. Allotype female, larval and pupal skins on slide (3003), right hindleg missing; ibid., 03 Jul 1940. Central Colection. Zavortink 1979: 12 (from genus Trichoprosopon).

- walcotti (Lane and Cerqueira, 1942). Runchomyia (Ctenogoeldia). Holotype female, right wing on slide (1927), right midleg missing; Caravelas, Bahia, Brazil; NC Davis coll., Jan 1931. Central collection. Zavortink 1979: 12 (from genus Trichoprosopon).

\section{Genus Sabethes Robineau-Desvoidy}

- batesi Lane and Cerqueira, 1942. Sabethes (Sabethes). Holotype male; genitalia on slide (2193); Tinguá, RJ, Brazil; Lane coll.; Jul 1940. Central collection.

- fabricii Lane and Cerqueira, 1942. Sabethes (Peytonulus). Holotype male; genitalia, larval and pupal skins on slides (2752, 2753); label data: "Angra dos Reis, RJ, Brazil; L Whitman coll.; Sept 1940". Allotype female (22447), larval and 
pupal skins on slide (2370); Iguassú, RJ, Brazil; ibid. Central collection. Harbach 1991: 1 (from subgenus Sabethinus).

- lutzianus Lane and Cerqueira, 1942. Sabethes (Peytonulus). Holotype male, Iguassú, RJ, Brazil; L Whitman coll., Aug 1940; larval and pupal skins and genitalia on slides $(2498,2751)$. Allotype female, ibid.; larval and pupal skins on slide (2998). Central collection. Synonym of Sabethes (Peytonulus) identicus Dyar and Knab, 1907. Lane 1951: 335 (syn.). Harbach 1991: 1 (from subgenus Sabethinus).

- lutzii Theobald, 1903. Sabethes (Sabethes). Holotype possibly represented by slide (1278) of wing, marked "da Coll. do Dr. Lutz/XI-930/ C.L."; type locality, Manaus, Amazonas, Brazil. Costa Lima collection. Belkin 1968: 31 (nomen dubium).

- petrocchiae (Shannon and del Ponte, 1928). Sabethes (Davismyia). Paratype female (2301); Raco - Tucuman, Argentina; RC Shannon col, 13 Feb 1927. Costa Lima collection. Del Ponte and Cerqueira 1938: 227 (ressurected from syn. with arthrostigma). Harbach and Peyton 1991: 157 (from genus Wyeomyia).

- purpureus Peryassú, 1908. Sabethes (Sabethes). Lectotype (vial 505); head and thorax on 1 pin, leg on point on another pin, wing on slide (1092); Juiz de Fora, Minas Gerais, Brazil; A Neiva coll., 28 Oct 1907. Homonym of Sabethes (Sabethes) purpureus (Theobald, 1907). Costa Lima collection. Belkin et al. 1971: 14 (lectotype desig.). Sabethes remipusculus Dyar, 1924: 100 (nom. nov. for purpureus Peryassú, non Theobald 1907).

- soperi Lane and Cerqueira, 1942. Sabethes (Peytonulus). Holotype male; label data: Iguassú, RJ, Brazil; L Whitman coll., Aug 1940; larval and pupal skins and genitalia on slides (2742, 2743). Allotype female; ibid.; pupal skin on slide (3010). Central collection. Harbach 1991: 1 (from subgenus Sabethinus).

- whitmani Lane and Cerqueira, 1942. Sabethes (Peytonulus). Holotype male, Santa Tereza, Espírito Santo, Brazil; L Whitman coll., May 1940; larval and pupal skins and genitalia on slides (2074, 2757). Allotype female; larval and pupal skins on slide (2758); ibid. Central collection. Harbach 1991: 1 (from subgenus Sabethinus).

\section{Genus Shannoniana Lane and Cerqueira}

- longipalpis Lutz, 1905. Shannoniana. Syntypes: 1 male (2635) and 3 females (2636-2638), possibly male (2635); Saúde, São Paulo, SP, Brazil; 05 Apr 1905. Central collection; genitalia on slide (1315). Costa Lima collection. Synonym of Trichoprosopon (Shannoniana) fluviatilis (Theobald, 1903). Lane and Cerqueira 1942: 503 (syn.). Zavortink 1979: 11 (from genus Trichoprosopon).

\section{Genus Trichoprosopon Theobald}

- brevipes (Costa Lima, 1931). Trichoprosopon. Lectotype male (vial 462), right hindleg and all tarsomeres missing except those of right midleg, genitalia and pupal skin on slides $(1028,1029)$; Rio de Janeiro, Brazil; José Francisco Ladeira (da Silva) coll., 03 Jan 1930. Costa Lima collection. Belkin et al. 1971: 9 (lectotype desig.).

- castroi Lane and Cerqueira, 1942. Trichoprosopon. Holotype male, genitalia on slide (1923); Teresópolis, Rio de Janeiro, Brazil; RC Shannon coll., Apr 1938. Allotype female, ibid. Central collection.

- evansae Antunes, 1942. Trichoprosopon. Holotype male, genitalia on slide (3000); El Caibe, Meta, Restrepo, Colombia; PCA Antunes coll., Dec 1934. Central collection.

- lanei (Antunes, 1937). Trichoprosopon. Holotype male (2299), genitalia on slide (2654); Caney, Restrepo, Meta, Colombia; PCA Antunes coll., 07 Feb 1935. Allotype female (2300); Vega Grande, Restrepo, Colombia; PCA Antunes coll., 23 Mar 1935. Costa Lima collection.

- obscurum Lane and Cerqueira, 1942. Trichoprosopon. Holotype male, genitalia on slide (1973); Mangaratiba, RJ, Brazil; RC Shannon coll., Apr 1938. Allotype female; ibid. Central collection.

- simile Lane and Cerqueira, 1942. Trichoprosopon. Holotype male, genitalia on slide (1203), left foreand right hindlegs and right foretarsi missing; Campos do Jordão, São Paulo, Brazil; F Lane coll., Dec 1935. Allotype female; ibid. Central collection.

- soaresi Lane and Cerqueira, 1942. Trichoprosopon. Holotype male, pupal skin and genitalia on slides (2711, 2755); São João de Petrópolis, Santa Teresa, Espírito Santo, Brazil; L Whitman coll., May 1940. Allotype female, pupal skin on slide (2712); ibid. Central collection.

\section{Genus Wyeomyia Theobald}

- airosai Lane and Cerqueira, 1942. Wyeomyia. Holotype male (4283), larval and pupal skins and genitalia on slides $(2271,2284)$, right fore- and hindlegs missing; Santa Tereza, Espírito Santo, Brazil; Serv. Nac. de Febre Amarela coll., May 1940. Allotype female (4301), larval and pupal skins, maxilla and mandible of larva on slide (2281); ibid. Central collection.

- alani Lane and Cerqueira, 1957. Wyeomyia (Antunesmyia). Holotype male, larval and pupal skins and genitalia on slides $(2389,2400)$; [Rio 
de Janeiro, Brazil]; L Whitman coll., Oct 1940. Allotype female, larval and pupal skins on slide (2346), tibiae and tarsi of midlegs missing; ibid.; Jul 1938. Central collection. Lane and Cerqueira, 1957: 244 (nom. nov. for rooti Lane and Cerqueira 1942, non Del Ponte 1939).

- arthrostigma (Lutz, 1905). Wyeomyia (Wyeomyia). Lectotype female (vial 927); Brazil; A Lutz coll. Costa Lima collection. Belkin et al. 1971: 10 (lectotype desig.).

- brucei Del Ponte and Cerqueira, 1938. Wyeomyia. Holotype female (2211); Cuiabá, Mato Grosso, Brazil; Gastão Cesar coll., Jun 1935. Costa Lima collection. Allotype male, genitalia on slide (2031), right hindleg missing; Fazenda Martinez - Cachoeira, Rio de Janeiro, Brazil; Del Ponte and Cerqueira coll., May 1938. Central collection. Synonym of Wyeomyia (Dendromyia) personata (Lutz, 1904). Costa Lima 1943: 306 (syn.).

- cesari Del Ponte and Cerqueira, 1938. Wyeomyia. Holotype female (2209); Cuiabá, Mato Grosso, Brazil; Gastão Cesar coll., Mar 1935. Costa Lima collection.

- delpontei Lane and Cerqueira, 1942. Wyeomyia. Holotype female; Cuiabá, Mato Grosso, Brazil; Feb 1935. Central collection. Synonym of Wyeomyia (Dendromyia) rooti (Del Ponte, 1939). Lane and Cerqueira 1957: 244 (syn.).

- finlayi Lane and Cerqueira, 1942. Wyeomyia. Holotype male, larval and pupal skins and genitalia on slides $(2260,2259)$ and right wing broken; Xerém, RJ, Brazil; Serv. Nac. de Febre Amarela coll., Jun 1940. Allotype female, larval and pupal skins on slide (2258), right fore- and midlegs missing; Tinguá, RJ, Brazil; Sept 1940. Central collection.

- forcipenis Lourenço-de-Oliveira and Silva, 1985. Wyeomyia. Holotype male (6125), larval and pupal skins, and genitalia on slides (5544, 5545, 5546); Granjas Calábria, Jacarepaguá, Rio de Janeiro, Brazil; R Lourenço-de-Oliveira coll., 08 Apr 1982. Allotype female (6126), larval and pupal skins on slide (5548); ibid.; R Lourençode-Oliveira coll., 11 Mar 1982. Costa Lima collection. Paratypes: 7 males and 2 females (66$69,152,185,197,591,593)$. Central collection.

- howardi Lane and Cerqueira, 1942. Wyeomyia. Holotype male, genitalia on slide (2299), all right legs, left midtarsomeres III-IV and left hindtarsomeres IV, V missing; Muriqueira, Bahia, Brazil; RC Shannon coll., 26 Apr 1929. Allotype female, right midleg and right hindtarsus missing; ibid. Central collection.

- kerri Del Ponte and Cerqueira, 1938. Wyeomyia. Holotype female (2210); Cuiabá, Mato Grosso, Brazil; Gastão Cesar coll., 15 May 1935. Costa Lima collection.
- knabi Lane and Cerqueira, 1942. Wyeomyia. Holotype male, genitalia on slide (2027) and left foretarsus missing; Fazenda Martinez Cachoeira, Rio de Janeiro, Brazil; Serv. Nac. de Febre Amarela coll., May 1938. Allotype female; Mangaratiba, RJ, Brazil; Jun 1938. Central collection.

- kummi Lane and Cerqueira, 1942. Wyeomyia (Cruzmyia). Holotype female (10140), right hindleg missing; Curralinho, Pará, Brazil; Serv. Nac. de Febre Amarela coll., Feb 1936. Central collection.

- limai Lane and Cequeira, 1942. Wyeomyia (Wyeomyia). Holotype male (12732), genitalia on slide (1528); Londrina, Paraná, Brazil; Serv. Nac. de Febre Amarela coll., Jan 1937. Allotype female (11628), left mid- and hindtarsi missing; ibid. Central collection.

- luteoventralis Theobald, 1901. Wyeomyia (Dendromyia). Paralectotype male (1475), larval and pupal skins and genitalia on slide (1475); Belém, State of Pará, Brazil; MA Motta coll., 28 Mar 1993. Central collection. Motta and Lourenço-de-Oliveira 1995: 375 (subgenus Dendromyia).

- lutzi (Costa Lima, 1930). Wyeomyia (Wyeomyia). Syntypes: 1 male (513) and 2 females $(472,514)$, larval and pupal skins and genitalia on slides (1058, 1066, 1084, 1096-1101, 1103, 1104 1118, 1119, 1171-1176); Alto da Boa Vista, Tijuca, Rio de Janeiro, Brazil; CA Campos Seabra coll., 28 Feb, 20 Apr 1930. Costa Lima collection.

- oblita (Lutz, 1904). Wyeomyia (Wyeomyia). Syntype male (vial 1544), with associated wing and genitalia on slides $(4599,4600)$, all legs broken except the left foreleg; Pacaembú, SP, Brazil; A Lutz coll., 11 Oct 1904. Costa Lima collection.

- pintoi (Costa Lima, 1930). Wyeomyia (Wyeomyia). Holotype female (vial 618), proboscis, palpi and antennae broken; Dr Olímpio da Fonseca's farm, Mendes, RJ, Brazil; 26 Jun 1930. Paratypes: 3 males (vials 616,617), larval and pupal skins on slides (1146-1150) and genitalia (616) on slides (1184-1187), one of them without genitalia; ibid. Costa Lima collection. Synonym of Wyeomyia (Wyeomyia) oblita (Lutz, 1904). Lane and Cerqueira, 1942: 562 (syn).

- rooti (Del Ponte, 1939). Wyeomyia. Lectotype female, same specimen as the holotype of delpontei Lane and Cerqueira, 1942; Cuiabá, Mato Grosso, Brazil; Feb 1935. Costa Lima collection. Belkin et al. 1971: 11 (lectotype desig.).

- sabethea Lane and Cerqueira, 1942. Wyeomyia (Wyeomyia). Allotype female; Teresópolis, RJ, Brazil; L Whitman coll., Apr 1938. Holotype male, larval and pupal skins, genitalia and wing 
on slides (2401, 3008, 3009), right midleg missing; label data: "Rio de Janeiro, Brazil; L Whitman coll., Nov 1940". Central collection.

- serrata (Lutz, 1905). Wyeomyia (Wyeomyia). Lectotype male, one leg, genitalia and wing on slides (1167-1170), right mid- and hindlegs missing; R. Frio, Pindamonhangaba, São Paulo, Brazil. Costa Lima collection. Belkin et al. 1971: 10 (lectotype desig.).

- shannoni Lane and Cerqueira, 1942. Wyeomyia. Allotype female; Mangaratiba, RJ, Brazil; RC Shannon coll., Apr 1938. Holotype male, genitalia on slide (2034); data from label: "Petrópolis, RJ, Brazil”; ibid. Central collection.

- staminifera Lourenço-de-Oliveira, Motta and Castro, 1992. Wyeomyia. Holotype male (1264), larval and pupal skins and genitalia on slide (1264); Ariquemes, Rondônia, Brazil; TF da Silva coll., 23 Jul 1987. Allotype female (1133), larval and pupal skins on slide (1133); R Lourenço-de-Oliveira coll., 28 Mar 1987. Paratypes: 6 males $(1131,1349,1365,1366$, 1369, 1370) and 1 female (1121). Central collection.

- tarsata Lane and Cerqueira, 1942. Wyeomyia (Exallomyia). Holotype male (8155), genitalia on slide (2079); Anápolis, Goiás, Brazil; Veiga and O Verano coll., Sept 1936. Allotype female (8155), mid- and hindlegs missing; ibid. Central collection. Harbach and Peyton 1992: 92 (from Subgenus Dendromyia).

- undulata Del Ponte and Cerqueira, 1938. Wyeomyia. Holotype female (2213); Cuiabá, Mato Grosso, Brazil; Gastão Cesar coll., Feb 1935. Costa Lima collection. Allotype male, genitalia on slide (2245); Mangaratiba, RJ, Brazil; RC Shannon coll., Apr 1938. Central collection.

Tribe Uranotaeniini

Genus Uranotaenia Lynch Arribálzaga

- noctivaga Neiva and Pinto, 1922. Uranotaenia (Uranotaenia). Holotype female (2025); Gávea, Rio de Janeiro, Brazil; Arthur Neiva coll., 1922. Cesar Pinto collection. Synonym of Uranotaenia (Uranotaenia) nataliae Lynch-Arribálzaga, 1899. Lane, 1953 (syn.).

Subfamily Toxorhynchitinae

Genus Toxorhynchites Theobald

- bambusicola (Lutz and Neiva, 1913). Toxorhynchites (Lynchiella). Syntypes: 7 males (533, 543, 1575-1579) and 2 females (544, 1580); Petrópolis, RJ, Brazil; JG Foetterle et al. coll. Costa Lima collection.

- catharinensis (Costa Lima, Guitton and Ferreira, 1962). Toxorhynchites (Ankylorhynchus). Holotype female (5553), pupal skin on slide (4869); Brusque, Santa Catarina, Brazil; 01 Jan 1951.
Allotype male (5549), genitalia, larval and pupal skins on slides (4862-4864); ibid., Apr 1951. Paratypes: 2 males $(5551,5554)$ and 2 females (5550, 5552), genitalia on slides $(4866,4861$, $4865,4868)$ and pupal skin of female (5552) on slide (4867); ibid. Costa Lima collection.

- fluminensis (Peryassú, 1908). Toxorhynchites (Lynchiella). LECTOTYPE by present designation, male (vial 535), genitalia on slide (1128); Rua Conde de Bonfim, Tijuca, Rio de Janeiro, Brazil; C Chagas coll., 16 Apr 1907. Costa Lima collection. Synonym of Toxorhynchites theobaldi (Dyar and Knab, 1906). Costa Lima 1931: 312 (syn.).

- posticatus (Lutz and Neiva, 1913). Toxorhynchites (Lynchiella). Syntypes: 2 females (549, 550 ), right hindtarsomeres of female (550) missing; Petrópolis, RJ, Brazil; 1912. Costa Lima collection. Synonym of Toxorhynchites (Lynchiella) theobaldi (Dyar and Knab, 1906). Lane, 1953 (syn.).

- pusillus (Costa Lima, 1931). Toxorhynchites (Lynchiella). Syntypes: 1 male (vial 541) and 1 female (vial 526), larval and pupal skins on slides (1121, 1135, 1102, 1116); Alto da Boa Vista, Tijuca, Rio de Janeiro, Brazil; CA Campos Seabra coll., 20 Apr 1930. Costa Lima collection.

- rizzoi (Deus Palma and Galvão, 1969). Toxorhynchites (Lynchiella). Holotype female (6112), antennae missing and left midleg broken, larval and pupal skins on slides (5535); Goianópolis, Goiás, Brazil. Costa Lima collection.

\section{DISCUSSION}

The only remaining male from the unspecified number of males, females and larvae utilized by Peryassú (1908) in the original description of Toxorhynchites fluminensis is herein designated as the lectotype. It agrees in all pertinent characters and locality specified in the species original description.

Belkin et al. (1971) designated lectotypes for both Anopheles cuyabensis and Anopheles rondoni, being wings of adults apparently lost, mounted together on the same slide (no. 403). Nevertheless we have found at IOC specimens of each of these species, labelled "type" by the authors (A Neiva \& C Pinto), and having all pertinent characters and localities specified in the respective original species description. These specimens are here labelled as paralectotypes.

The holotype of six species previously considered lost or of unknown location by Belkin et al. (1971) and Knight and Stone (1977), were found at IOC: Psorophora chiquitana, Psorophora circunflava, Psorophora melanota and Psorophora lanei, Uranotaenia noctivaga and Wyeomyia brucei. 
Belkin et al. (1971) supposed that the two demaged females (no. 2048, vial 1060), from Duas Barras, State of Minas Gerais, Brazil (wing on slide 2347), Costa Lima collection of Psorophora (Grabhamia) scutipunctata (Lutz and Neiva, 1911) presently at IOC could be syntypes. However, these specimens were not collected at the type locality and were not marked as "type" by the authors.

Belkin et al. (1971) designated a male in the U.S.N.M. as the lectotype for Phoniomyia edwardsi. A single female labelled by J Lane and NL Cerqueira as the "allotype" of this species was found at IOC, which is here labelled as a paralectotype.

The type material of Anopheles intermedius (Peryassú, 1908), Culex fasciolatus (Lutz,1904), Culex theobaldi (Lutz, 1904), Trichoprosopon pallidiventer (Lutz, 1905), Trichoprosopon humboldti Lane and Cerqueira, 1942, Trichoprosopon compressum Lutz, 1905, Wyeomyia rooti (Del Ponte, 1939 [possibly in CP René Rachou, Belo Horizonte, Brazil]) and Wyeomyia subcomplosa (Del Ponte, 1939 [possibly in CP René Rachou]) were not found after exhaustive search in the collection at IOC. The specimens previously listed or suggested as possibly being type material of Anopheles lineatus (Lutz, 1905), Anopheles parvus (Chagas, 1907) and Anopheles pseudomaculipes (Peryassú, 1908) by Belkin et al. (1971) and Knight and Stone (1977) are not from the respective type localities. They are without data, and because they have questions marks on the slides and vials prepared by Costa Lima nearly thirty years after the original description, there is no justification for affording them type status.

\section{REFERENCES}

Belkin JN 1968. Mosquito studies (Diptera, Culicidae). IX. The type specimens of New World mosquitoes in European museums. Contrib Am Entomol Inst 3: 1-69.

Belkin JN, Schick RX, Heinemann SJ 1971. Mosquitoes studies (Diptera, Culicidae). XXV. Mosquitoes originally described from Brazil. Contrib $\mathrm{Am}$ Entomol Inst 7: 1-64.

Correa RR, Ramalho GR 1956. Revisão de Phoniomyia Theobald, 1903 (Diptera, Culicidae, Sabethini). Folia clin biol S Paulo 25: 1-176, illus.

Costa Lima A 1931. Sobre as espécies de Megarhinus do Brasil (Diptera, Culicidae). Mem Inst Oswaldo Cruz 25: 307-315, 2 pls.

Costa Lima A 1935. Espécies de Taeniorhynchus (Taeniorhynchus) (Diptera, Culicidae). Mem Inst Oswaldo Cruz 30: 453-469, 10 pls.

Costa Lima A 1943. Notas sobre alguns sabetineos. Ann Acad bras Sci 15: 295-308.

Dyar HG 1924. A note on Sabethes Robineau-Desvoidy (Diptera, Culicidae). Insec Inscit menst 12: 97-100.
Gonçalves TCM, Almeida MD, Lent H, Jurberg J 1994. Levantamento dos exemplares tipos de Triatomíneos depositados na Coleção Entomológica do Instituto Oswaldo Cruz. Mem Inst Oswaldo Cruz 88: 327 333.

Harbach RE 1991. A new subgens of the genus Sabethes (Diptera, Culicidae). Mosq Syst 23: 1-9.

Harbach RE, Peyton EL 1991. Transfer of the subgenus Davismyia from Wyeomyia to Sabethes and description of the type species, Miamyia petrochiae (Diptera, Culicidae). Mosq Syst (1990) 22: 149159.

Harbach RE, Peyton EL 1992. A new subgenus of Wyeomyia (Diptera, Culicidae), with the reclassification and redescription of Wyeomyia (Davismyia) arborea, Wyeomyia (Dendromyia) tarsata and Sabethes (Sabethes) carrilloi. Mosq Syst (1991) 23: 92-109.

Kim KC 1976. The changing nature of entomological collections: uses, functions, growth and manangement. Entomologica Scandinavica 9 : 146177.

Knight KL, Stone A 1977. A Catalog of the Mosquitoes of the World (Diptera: Culicidae). 2nd edition. Thomas Say Found., Entomol. Soc Am, vol 6, XI + $611 \mathrm{p}$.

Lane J 1951. Synonymy of Neotropical Culicidae (Diptera). Proc ent Soc Wash 53: 333-336.

Lane J 1953. Neotropical Culicidae. 1112 pp., illus. São Paulo, Brazil.

Lane J, Cerqueira NL 1942. Os sabetineos da América (Diptera, Culicidae). Arch Zool S Paulo 3: 473849 , illus.

Lane J, Cerqueira NL 1957. The validity and change of name of two species of Wyeomyia (Diptera, Culicidae). Proc Entomol Soc Wash 59: 244.

Lent H 1972. Desenvolvimento da Entomologia no Instituto Oswaldo Cruz. Ci Cul, 24 : 1192-1200.

Motta MA, Lourenço-de-Oliveira R 1995. Wyeomyia luteoventralis Theobald, the type species of the subgenus Dendromyia Theobald (Diptera: Culicidae). Mem Inst Oswaldo Cruz 90: 375-385.

Peryassú AG 1908. Os Culicideos do Brasil. 407 pp. Instituto de Manguinhos, Rio de Janeiro, Brasil.

Pinto C 1939. Disseminação da malária pela aviação; biologia do Anopheles gambiae e outros anofelineos do Brasil. Mem Inst Oswaldo Cruz 4: 293-430, illus., 61 pls.

Rosa-Freitas MG, Deane LM 1989. The neotype of Anopheles albitarsis (Diptera: Culicidae). Mem Inst Oswaldo Cruz 84: 289-302.

Sirivanakarn S 1983. A review of the systematics and a proposed scheme of internal classification of the New World subgenus Melanoconion of Culex (Diptera, Culicidae). Mosq Syst (1982) 14: 265-333.

Zavortink TJ 1968. Mosquito Studies (Diptera, Culicidae). VIII. A prodrome of the genus Orthopodomyia. Contrib Am Entomol Inst 3: 221.

Zavortink TJ 1979. Mosquito studies (Diptera, Culicidae) XXXV. The new sabethine genus Johnbelkinia and a preliminary reclassification of the composite genus Trichoprosopon. Contrib Am Entomol Inst (Ann Arbor) 17: 1-61. 